\title{
Experimental characterization of natural fibre-soil interaction: lessons for earthen construction
}

\author{
Alessandra Vincenzini (i) C Charles E. Augarde $(\mathbb{D} \cdot$ - Massimiliano Gioffrè $\mathbb{C}$
}

Received: 2 May 2020/ Accepted: 8 April 2021/Published online: 3 May 2021

(C) The Author(s) 2021

\begin{abstract}
Earthen construction materials are the subject of renewed interest due to the rising alarm about environmental pollution from the construction industry. Current research efforts are focused on improving the mechanical properties of earthen materials to make them modern and competitive. To increase strength and improve ductility fibres can be added to the soil mixture and if natural fibres are used one achieves stabilisation in an environmentally friendly way. Several previous studies have dealt with the behaviour of this composite material at a macroscopic level and on the general interaction between fibres and soil, but there is little published research on the interfacial mechanical interaction between natural fibre reinforcement and a soil matrix which is key to the former. This paper attempts to fill this gap by presenting and discussing laboratory results from a large campaign of pull-out tests conducted on composite earthen samples. The variables investigated
\end{abstract}

A. Vincenzini · M. Gioffrè ( $₫)$

Department of Civil and Environmental Engineering, University of Perugia, Via G. Duranti, 93, 06125 Perugia, Italy

e-mail: massimiliano.gioffre@unipg.it

A. Vincenzini

e-mail: alessandra.vincenzini@unipg.it

C. E. Augarde

Department of Engineering, Durham University, South

Road Durham, Durham DH1 3LE, UK

e-mail: charles.augarde@durham.ac.uk here are the nature of the fibres (i.e. single or collections twisted together) and the use of fibre treatments such as PVA glue and baking soda. In the study both fibre-soil failure and soil-soil failure are investigated and the results lead to conclusions as to appropriate use of fibres to reinforce earthen construction materials.

Keywords Earthen materials - Fibre reinforcement · Natural fibre · Pull-out test · Jute fibre - Sustainability · experimental charachterization

\section{Introduction}

Earth has been used as a building material since prehistoric times and even today it is estimated that at least a third of the world's population live in houses built with earth [1]. One of the oldest settlements found has been linked to the Phoenicians [2], and the oldest group of houses surviving in Europe is at Skara Brae in Orkney, Scotland [3]. This Scottish settlement was built two and a half thousand years before the Great Wall of China, parts of which represent one of the largest rammed earth construction projects ever undertaken [4]. At various times in the modern era, and especially since the 1970 s there has been renewed interest in this ancient method of building. It is partly a result of the increasing awareness of the role that 
construction, one of the most resource-hungry and least sustainable industries in the world, must play to reduce global warming and $\mathrm{CO}_{2}$ emissions. Tables 1 and 2 summarise the data concerning resource usage and environmental impact of the entire construction process, provided by the United Nations Environment Programme (UNEP). Energy consumption can be reduced by using low impact materials, locally available, which carry low embedded energy. Earth, in this context, potentially represents a valuable sustainable material resource: recyclable, non-polluting and in plentiful supply in many areas of the world.

The earthen construction historical record presents an opportunity to learn from the traditional building methods employed in the past, in order to make revisions for efficient sustainable use today and in the future. However, for full acceptance, increased scientific research is needed to promote the use and the development of earthen materials and to allow the development of design codes as is the case for conventional building materials. Different approaches and future challenges have been presented by Augarde et al. [5] in a recent conference paper.

One example of earthen construction is Rammed Earth which is a construction technique involving the compaction of a soil mixture into temporary formwork. The mix used is a combination of inorganic subsoil and water, to which it is possible to add different additives and stabilisers. The soil must contain a certain particle size distribution (gravel, silt, sand and clay) and it must have specific properties such as plasticity, compressibility and cohesion [4]. The strength of rammed earth largely depends on the uniformity and consistency of the mixture achieved. Once at the optimum moisture content, determined in

Table 1 Estimates of global resources used in buildings

\begin{tabular}{ll}
\hline Resource & $\%$ \\
\hline Energy & $45-50$ \\
Water & 50 \\
Materials for buildings and roads & 60 \\
Agricultural land loss to buildings & 80 \\
Timber products for construction & 60 (90\% of hardwoods) \\
Coral reef destruction & 50 (indirect) \\
Rainforest destruction & 25 (indirect) \\
\hline
\end{tabular}

Table 2 Estimates of global pollution attributed to buildings

\begin{tabular}{ll}
\hline Pollution & $\%$ \\
\hline Air quality & 23 \\
Climate change gases & 50 \\
Drinking water & 40 \\
Landfill waste & 50 \\
Ozone depletion & 50 \\
\hline
\end{tabular}

various ways as discussed in [6], the mixture is placed into formwork layer by layer and compacted with rammers. The compression energy employed influences the soil density which also depends on the moisture content, composition and grading. Layers must be maximum $100 \mathrm{~mm}$ thick before ramming and compacting to $50-70 \mathrm{~mm}$. Skilled workers are fundamental to ensure good durability and resistance of rammed earth structures [7].

The main limitations of unstabilised earthen materials (i.e. those containing just a soil mixture) concern its low strength and brittle behaviour. This paper is concerned with one means of improving these properties: the addition of fibres. This can provide improved ductility and increased strength, in addition to shrinkage prevention and limitation of crack propagation [8]. The work presented here complements and adds to previous studies [9-11] focussing on the interfacial mechanical interaction between natural fibre reinforcement and a soil matrix, an area with a very limited literature to date. The innovation of this study is given by the original experimental tests results, which provide significant information on the fibre-earth interaction. While much of the research carried out here has focussed on rammed earth, the findings are applicable more widely to other earthen construction materials.

\section{Stabilised rammed earth}

Traditional rammed earth construction, termed rammed earth or unstabilised rammed earth involves the use of clay as the only binder. If small quantities of other binders such as cement or lime are added to the soil mix it becomes stabilised rammed earth. Stabilisation can also be provided by adding natural or synthetic fibres into the soil mixture. Both approaches change the material properties, but in different ways. Soil is a non-homogeneous material whose properties 
depend on a large range of factors such as soil characteristics, particle size distribution, dry density and water content, [12]. Therefore a wide variation is observed in physical and mechanical performances of stabilised and unstabilised rammed earth. A brief review of stabilisation techniques is given below.

\subsection{Additives}

The most common stabilisation procedure involves adding small quantities of binders such as cement, pozzolana, lime, gypsum or bitumen to the soil mixture. Additives have been commonly used in vernacular constructions to improve workability and to protect the material from weathering effects [12]. The most common additive used in modern earthen construction is cement [13]. Research shows that cement improves the strength of rammed earth and the surface finish, reducing erosion and the effects of frost attack. Walker et al. [7] present advantages and disadvantages of using cement, explaining how the properties change and indicating the cement percentages recommended by previous studies. However, it is now widely accepted that the impact that cement has on the environment (arising largely from how it is made) is not desirable. Looking for additives with lower environmental impacts is therefore of some value. Examples include starches, such as rice or wheat rice, and lignosulfates such as resinous and oily liquids, both of which have been shown to improve binding qualities and durability [12]. Chang et al. [14] undertook an investigation of the durability improvement of Korean residual soil using treated biopolymers, i.e. Beta-glucan, Xanthan and Agar gums, added at $1 \%$ by mass to the soil. Results showed higher flexural strength using all additives. In particular, Xanthan gum and Agar gum achieved an improvement of $10 \%$ more than gypsum. Muguda et al. [15] also found Xanthan gum to provide considerable increases in compressive and tensile strengths of engineered soil mixes when used as a stabiliser. Haricane et al. [16] studied the effects of lime and natural pozzolana on the compaction and strength of a soft-clay soil. Test results showed that stabilised soil had improved compaction properties and shear and unconfined compressive strength with a combination of lime and natural pozzolana producing better results than lime or pozzolana alone. More recently, internationally renowned architects have designed rammed earth constructions using different stabilisation materials and techniques. Studio Blaanc designed a house in the middle of a vineyard in Portugal, adding layers of fibreglass mesh between layers of compacted earth to provide structural support. A coat of water glass and casein (a protein substance) was also added to protect the earth surface [17]. Herzog and De Mouron recently designed an impressive herb processing plant with rammed earth walls in the Swiss countryside mixing local earth with marl that had similar properties to lime [18].

\subsection{Fibre reinforced soil}

Soil reinforcement most commonly refers to a geotechnical technique to improve engineering characteristics such as shear strength, compressibility, density and hydraulic conductivity [19]. Fibres were commonly used in vernacular earth constructions as cheap and abundantly available filler materials, the most common being straw, but hemp, flax, sisal and jute were also used as they have similar properties. Fibres are still added to earth bricks especially Adobe, but less commonly appear in mortars. The biodegradation of natural fibres occurs under aerobic and anaerobic conditions determining their durability as reinforcement material. Treatment methodologies also affect longevity and reinforcement capabilities [20] Nowadays, fibre-reinforced soil usually refers to the use of textile grids rather than random direct inclusion of fibres into the soil mix, which is the topic of this paper, although there is evidence of some increasing interest in the latter in recent years [21]. Discrete fibres provide tensile reinforcement across failure planes and can be added easily to the soil mix [22]. However, the literature is confusing in some areas. For instance, some papers report decreases in unconfined compressive strength for earth bricks reinforced with fibres $[23,24]$ while others report strength increases [25, 26]. These differences may be due to the soil mixtures used and the amount of fibres added to the soil. Studies tend to show that unconfined compressive strength is increased for a low fibre content when the soil mixture contains a high proportion of clay, but decreases when the fibre content is high and the soil is mainly sandy [11]. Fibres appear to improve strength when applied at low concentrations but a threshold exists beyond which fibres begin to bunch together and their presence becomes deleterious to the soil properties 
[27]. Investigations into the shearing behaviour of earth samples reinforced with wool fibres have shown fibres to increase both the angle of friction and to decrease cohesion [28, 29]. An increase of flexural strength with the introduction of fibres is shown in the study of Millogo et al. [30] with a significant improvement of $0.6 \mathrm{MPa}$ compared to samples with no fibres.

The studies cited above indicate that adding fibres can lead to increases in strengths. Fibre stabilisation also prevents shrinkage, limiting crack propagation, and improves the ductile behaviour of earth materials, which may be important for earthquake-prone areas [31, 31, 32]. The key mechanism is a tensile reinforcement suggesting that understanding the bonding mechanisms between soils used for earthen construction and fibres is important $[32,33]$.

\section{Fibre-soil interaction}

The experimental findings described above clearly confirm that adding fibres to a soil mix changes the mechanical properties of the composite material via tensile strength enhancement. Within this context, fibre pull-out represents a central issue in understanding the factors affecting the interaction between fibres and soil [21]. Tang et al. [34] investigated the pull-out strength of polypropylene fibre reinforced soil finding that the interfacial residual strength increases with an increase in soil dry density, while it decreases with increasing water content. Cement was also added to the soil leading to a further significant improvement in the interfacial shear strength. Readle [9] analysed the effect of the variation of fibre length, water content and dry density using jute fibres. The results showed higher pull-out strengths were achieved by increasing sample length, decreasing water content and increasing dry density. Coghlan [10] used both natural and synthetic fibres and chose additives such as cement, lime and ash and found that that the use of natural fibres led to better results over synthetic ones and, in particular, jute fibres achieved the best adhesion with the soil mix.

If we consider pull-out via failure of the bond between the soil and the fibre then the likely influential features will be the form and roughness of the fibre surface and the stress normal to the fibre. Both of these are likely to be affected by dimensional changes of the soil, via shrinkage or swelling, and of the fibres, both of which can occur due to changes in moisture and temperature. Considering the fibres, changes in fibre diameter can occur during the curing stage of the soilfibre composite material, resulting in a poor interfacial bond. During the mixing stage, the hydrophilic nature of natural fibres can lead to water absorption which is then reversed during the drying stage. Fibres losing water shrink and voids can then form around the periphery of the fibre weakening the interfacial bond. This shrinkage and expansion mechanism of natural fibres is illustrated in Fig. 1.

\subsection{Treatments}

Since for sustainability purposes, one might wish to use natural rather than synthetic fibres, the water absorption issue becomes key and various workers have attempted to find a remedy. Ghavami et al. [35] used liquid bituminous materials such as piche and cipla to treat coconut and sisal fibres obtaining a significant reduction of the water absorption. However, bitumen reduces the strength of the soil-fibre bond. Alkali treatment of fibres leads to a change in surface roughness of the material due to chemical changes and the removal of lignin wax and oils covering the external surface of the fibre. Research presented in Nam et al. [36] showed that jute fibres used in composite materials, that had undergone alkali treatment exhibited lower water absorption compared to untreated fibres. Leaching effects due to chemical treatment also improved the fibre-matrix adhesion because of removal of natural and artificial impurities from the fibre surface as well as changing in the arrangement of units in the cellulose macromolecule. The loss of cellulose also increased the maximum flexural strain of the fibre [37].

In the study presented below, we add to the work described above, investigating the pull-put strength of natural fibres used as soil reinforcement, particularly

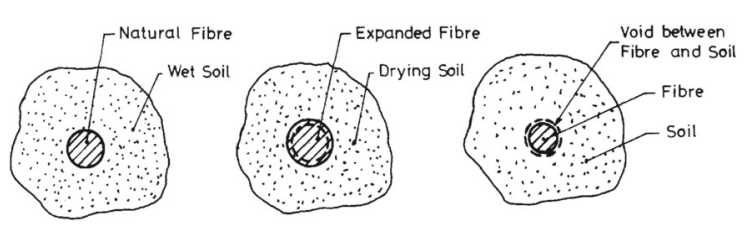

Fig. 1 Interaction of natural reinforcing fibre and drying soil [35] 
for rammed earth, making use of a number of novel additives and treatments.

\section{Pull-out experimental tests}

Jute fibres were chosen to allow comparisons with findings reported by Coghlan [10] and to remain coherent with the environmentally friendly characteristics of earthen materials. The jute fibres used in the pull-out tests had a mean ultimate tensile strength and mean Young modulus equal to $95.5 \mathrm{MPa}$ and 730 $\mathrm{MPa}$, respectively.

Three main variables were chosen: the number of fibres in a single sample, the twist of a group of fibres and thirdly, different treatments. Increasing the number of fibres allows investigation of the influence of fibre area to the pull-out strength. Investigating twist explores the role of the fibre shape in the adhesion properties. Fibre treatments should improve the bonding between the fibres and the soil matrix and also reduce the water absorption capacity. In this work the treatments chosen were PVA glue and baking soda. Cylindrical earthen samples were prepared with one or more embedded jute fibres (as in the experimental work described in [11]). The fibres were both in a wet and dry state before embedment in the soil matrix depending on the specific test as it will be described in the following sections. The embedment length of the fibre in the soil matrix corresponded to the whole earthen sample length (i.e. $50 \mathrm{~mm}$, Fig. 3a).

A preliminary test was carried out to evaluate the water absorption of untreated jute fibres. Following the test procedure proposed by Ghavami et al. [35], fifteen portions of jute fibres, $100 \mathrm{~mm}$ in length, were immersed in water at room temperature for eighteen days. Every $24 \mathrm{~h}$, the weight of each soaked fibre was recorded to obtain the water absorption percentage $(W)$ expressed as

$$
W=\frac{P_{\mathrm{h}}-P_{\mathrm{d}}}{P_{\mathrm{d}}}
$$

where $P_{\mathrm{h}}$ is the soaked weight and $P_{\mathrm{d}}$ the air dried weight. Fig. 2 shows the resulting water absorption variation with soaking time for the material used here compared with the results for coconut and sisal fibres reported in Ghavami et al. [35].

The jute fibre shows a trend similar to the other fibres, absorbing water rapidly during the first $24 \mathrm{~h}$.

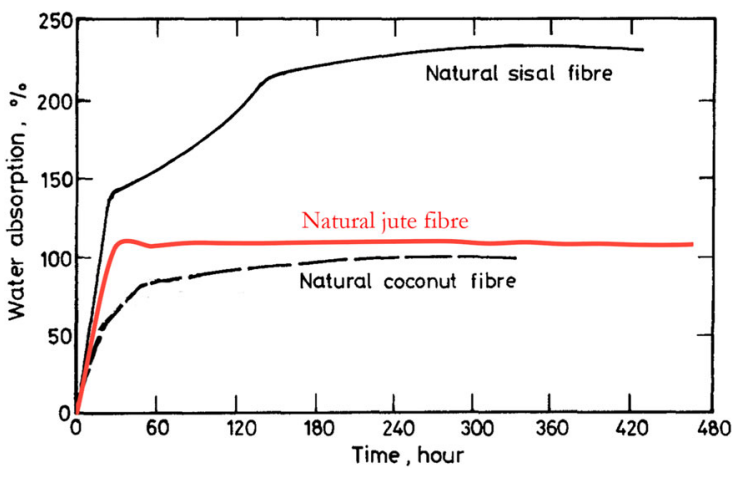

Fig. 2 Comparison of water absorption capacity in three natural fibres: figure adapted from [35]

After that, and unlike the other fibres, a stable value is reached around $100 \%$.

\subsection{Soil mix}

The soil mix chosen was an engineered mix to aid repeatability and contained $30 \%$ clay, $60 \%$ sand and $10 \%$ gravel. It is classified as 30:60:10 in the system of Smith and Augarde [38], and matches the mix used in other studies $[9,10]$. This mix achieves high unconfined compressive strength (UCS) and high dry density following natural drying as observed in other studies [39]. The size chosen for the samples meant that adding a gravel fraction could cause issues with repeatability and affect test results. It was deemed appropriate therefore to rearrange the soil mix to $63 \%$ sand and $37 \%$ clay. The optimum water content was determined via the Proctor test to be $11 \%$, in agreement with [10]. The soil mix was prepared by stirring together the right amount of dry clay and sand until a uniform grain distribution had been achieved, then the water was added gradually. The mixture was left to stand for 24 hours in a sealed bag to allow the equilibration of water content by the clay [4] and to achieve uniform distribution throughout the soil [40].

\subsection{Earthen samples}

Samples were made via a novel procedure developed previously by Readle [9] using a mould, plunger and baseplate as shown in Fig. $3 b$.

The mould is of a hollow cylindrical shape, $150 \mathrm{~mm}$ in length with an external diameter of $50 \mathrm{~mm}$ and an internal diameter of $27 \mathrm{~mm}$. The 

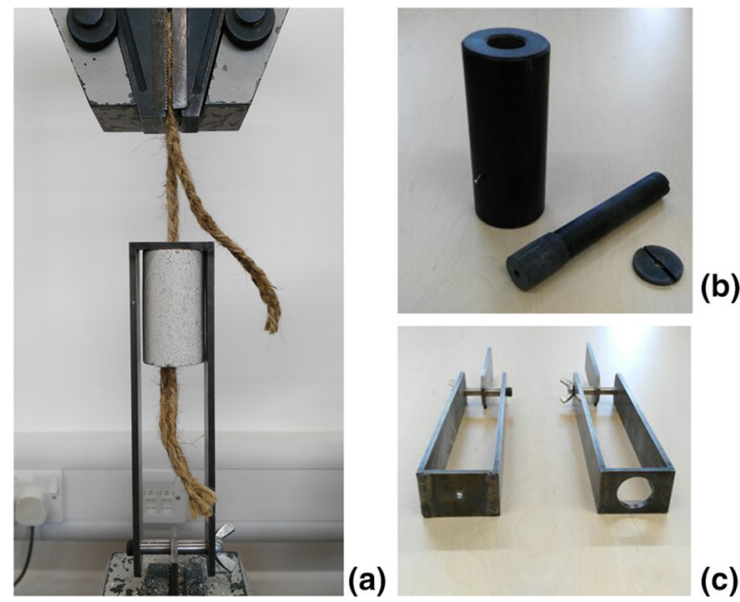

Fig. 3 Test set-up

plunger has a hole along its entire length to accommodate the fibre and the baseplate fits into one side of the mould and has a hole through which the fibre passes. To make samples, the soil mix was placed into the mould, the fibre passed through the plunger and the baseplate, tied off on one side of the mould and put under tension, thus ensuring that it remained in a central position during the static compaction process. The compaction load was applied uniformly to avoid stress concentrations and after compaction, samples were left to air dry for $24 \mathrm{~h}$ prior to testing. A visual inspection of every sample was made to guarantee the absence of defects or cracks and the attainment of a homogeneous texture. Figure 4 shows some of the cylindrical samples.

\subsection{Jute fibre treatments}

The jute fibres used in this study were treated with PVA glue and baking soda solutions. Baking soda $\left(\mathrm{NaHCO}_{3}\right)$ is mildly alkaline as compared to sodium hydroxide $(\mathrm{NaOH})$ used in Nam et al., [36] and

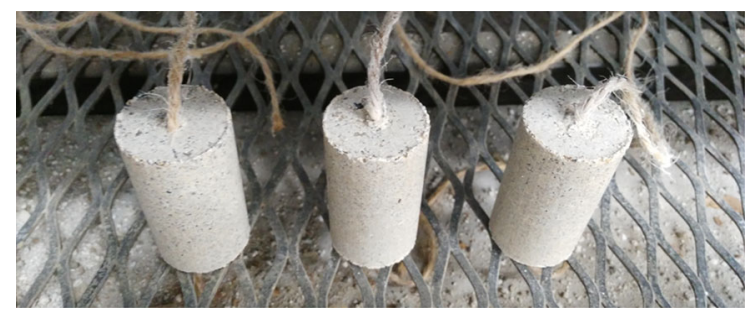

Fig. 4 Cylindrical earthen samples therefore perhaps more in keeping with health and safety considerations. PVA glue was chosen as a widely available product of similar nature to natural binders as Beta-glucan, Xanthan and Agar gums, mentioned in Sect. 2.1. Solutions of different concentrations (\% by mass) of both treatments were made somewhat tentatively due to the lack of similar experiments in this field. For the glue two solutions were used: $50 \%$ glue and $20 \%$ glue by mass. For the baking soda treatment the two solutions were $9 \%$ and $50 \%$ baking soda by mass. All treated fibres were first soaked for $3 \mathrm{~h}$, as suggested in [36] then, some fibres were left to dry out and others were used, still wet, in the earthen samples. For the fibres treated with the $50 \%$ baking soda solution, the obtained samples were allowed to dry out for two days. Fibres were then washed thoroughly with water and left to dry for a further two days to remove any trace of solution. After treatment with the baking soda, despite it being a weak alkali, fibres changed colour and were rougher to the touch.

\subsection{Test arrangement}

Samples judged suitable were subjected to pull-out tests on a Lloyds tensile testing machine. A proper restraint designed by Readle [9] was secured into the bottom clamp of the tester and then the sample was placed inside and the fibre (or fibres) exiting through the opening were secured to the top clamp at a distance of $50 \mathrm{~mm}$ as shown in Fig. 3a. Samples were loaded via displacement control at a rate of $1 \mathrm{~mm} / \mathrm{min}$ and the pull-out force and corresponding displacement were recorded. The tests were carried out for $40 \mathrm{~min}$ to fully explore peak and residual pull-out forces and displacements. Two types of failure were investigated: termed "matrix" and "interface", to replicate the two possible types of failure that could occur locally in fibre-reinforced earth construction. Matrix failure occurs in the soil matrix and in that case a restraining cylinder with a larger hole was used to allow displacements of the earth surrounding the fibres (Fig. 3c). Interface failure occurs when the bond between soil and fibre is broken, therefore to promote this in the tests, the sample is restrained close to the fibre circumference (Fig. 3c). To test samples with multiple fibres, different frames were designed to easily change the opening diameter. Three tests for each investigated parameter set were conducted. 


\section{Test results}

The results of the experimental tests are reported and compared in this section. In all tests peak and residual states were reached, where the peak value of force is the maximum reached early in the test, following which the force carried drops to a residual level which is maintained for a considerable displacement. The results are presented as force-displacement plots rather than stress-strain curves as the object here is to compare behaviour rather than provide accurate strength values. The same test procedure was used to obtain "matrix" and "interface" failure. Fibres failure was not observed in any of the presented pull-out tests.

\subsection{Samples with a single fibre}

Samples with single fibres were tested at the maximum dry density (MDD) of $1.96 \mathrm{Mg} / \mathrm{m}^{3}$ for both matrix and interface failure modes. Some samples with soil dry density of $2.00 \mathrm{Mg} / \mathrm{m}^{3}$ were also tested for the sake of comparison with other results already available. The plot in Fig. 7 shows the mean values for each mode and dry density considered indicating that samples at the dry density of $2.00 \mathrm{Mg} / \mathrm{m}^{3}$ reach higher maximum force values for both failure mechanisms compared to samples at the lower dry density which is no surprise. Higher peak and residual strength values are obtained for interface failure than for the matrix failure. These results match the findings in Readle [9] and Coghlan [10]. Samples were visually inspected after being tested and each exhibited a small groove around the fibre without clear signs of cracking when tested for the matrix failure mechanism, as shown in Fig. 5. Some samples tested for the interface failure mode were fully broken and others showed longitudinal cracking over the circular base as shown in
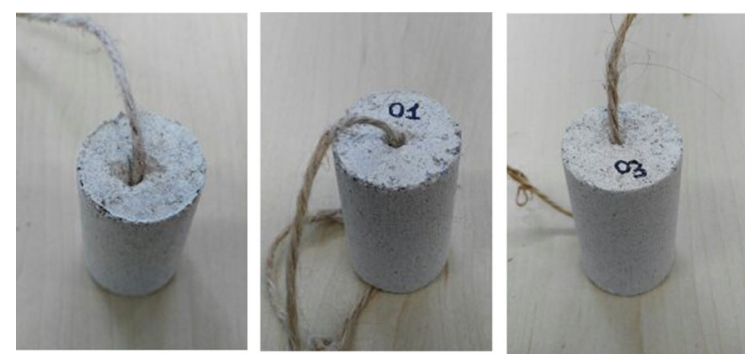

Fig. 5 Evidence of groove around the fibre; samples tested for the matrix failure mode

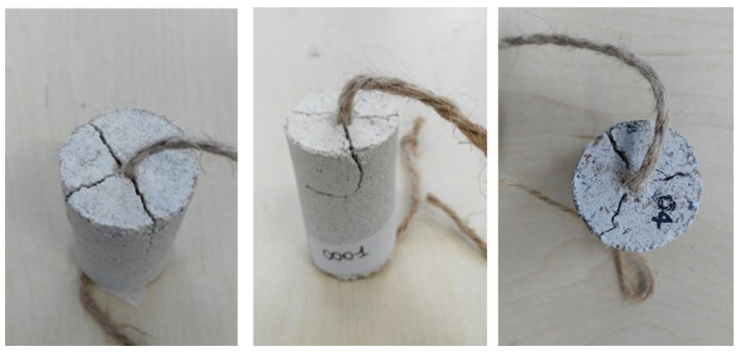

Fig. 6 Evidence of longitudinal cracks; samples tested for the interface failure mode

Fig. 6. The matrix failure results show that the weak link in the system is the fibre-soil bond. When the region around the fibre on the sample surface is restrained, in the interface tests, the capacity is increased due to the greater containment. Failure then only occurs when the tensile stresses induced at right angles to the fibre direction (due to a Poisson's ratio effect) crack the soil matrix.

\subsection{Samples with multiple fibres: no twist}

Samples with two and five twisted fibres were then tested. The results indicate that having two fibres does not lead to any significant change in capacity over a single fibre for both matrix and interface failure modes, as shown in Fig. 8. It appears that the soil matrix cannot achieve a strong bond around the entire circumference of a group of fibres and although the number of fibres is increased, the bond area is much the same hence there is no significant improvement. Testing on samples with five fibres was not successful and maybe this was too large a bundle to be supported in the sample size.

\subsection{Samples with multiple twisted fibres}

Samples were then tested with two and five twisted fibres. The results for interface failure were notable for being much more varied between tests in this case, therefore average values are not representative for this case and plots are shown for each sample in Fig. 9. Peak strength appears to be improved as compared to untwisted fibres but residuals are closer in agreement. It appears that the helical shape of twisted fibres may lead to an increase in the frictional bond with the soil matrix here and one can draw a parallel with the helices on reinforcing bars for concrete. The peak 

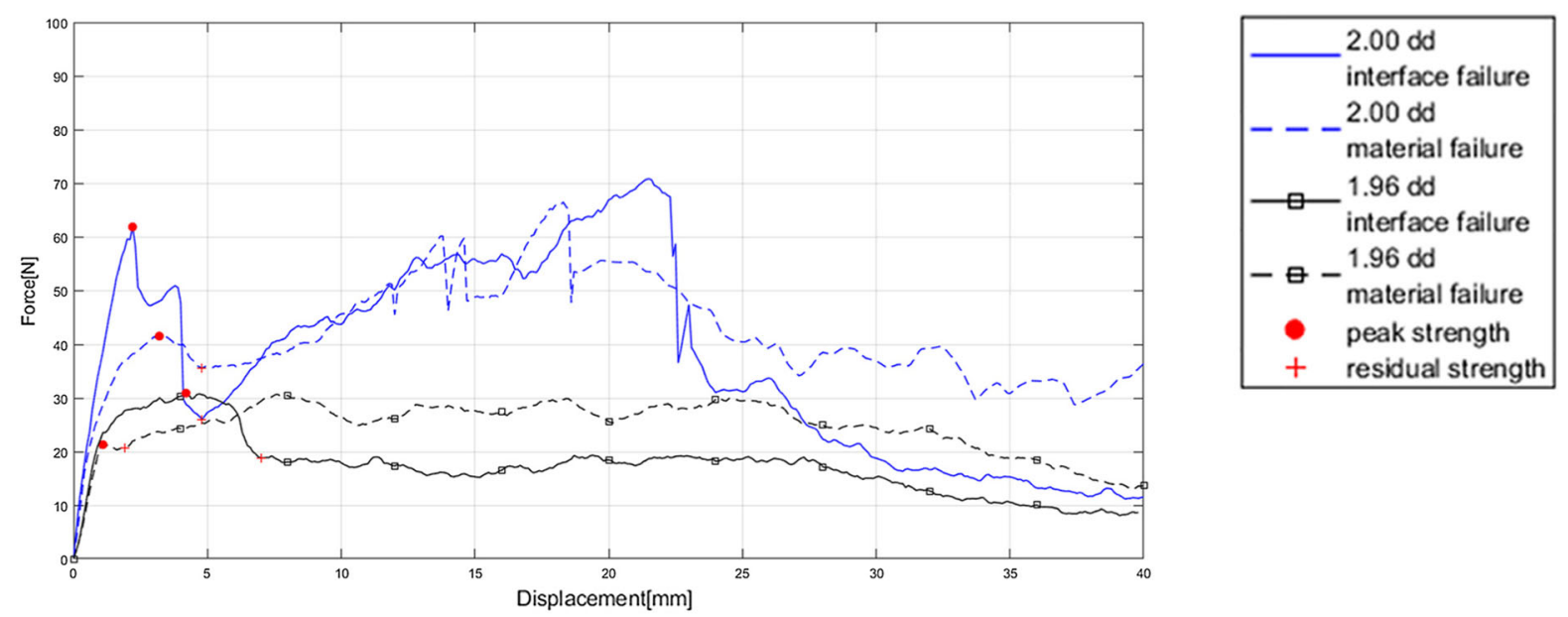

Fig. 7 Mean values results from tests with one fibre at a dry density of $2.00 \mathrm{Mg} / \mathrm{m}^{3}$, interface and matrix failure
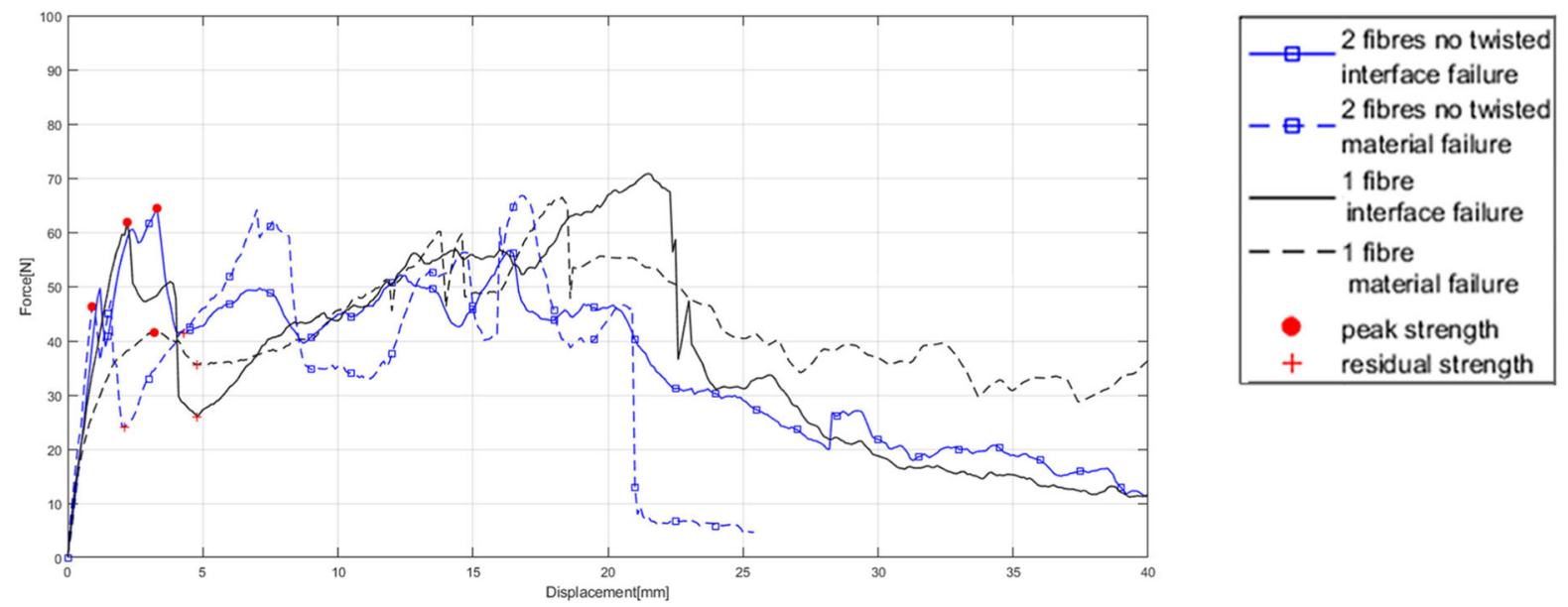

Fig. 8 Mean values results from tests with one fibre and two fibres with no twist at a dry density of $2.00 \mathrm{Mg} / \mathrm{m}^{3}$, interface and matrix failure

strengths for the matrix failure in these tests do not increase significantly as shown in Fig. 10, remaining close to the values found for tests with untwisted fibres. Nevertheless, of note is the fact that the behaviour is more ductile and the residual strength increases, especially for the case of two twisted fibres. Tests on samples with five fibres embedded show an increment of peak strength for the interface mode compared to samples with one fibre as shown in Fig. 11. That increment is probably not as much as it would be if the ratio between the surface occupied by the samples and the surface occupied by the fibres was higher, as already discussed in the previous section for samples tested with five untwisted fibres. The plots also show that the failure was achieved in a more ductile manner and residual strength was increased.

\subsection{Fibres treated with PVA glue}

As indicated above, samples were treated with PVA glue and some were left to dry while others were used to make samples when still wet (see Sect. 4.3). Figure 12 shows results for both cases showing that samples with fibres allowed to dry out reach very low peak strength values as compared to the wet tests. It is likely that when the glue is dry it does not react with 

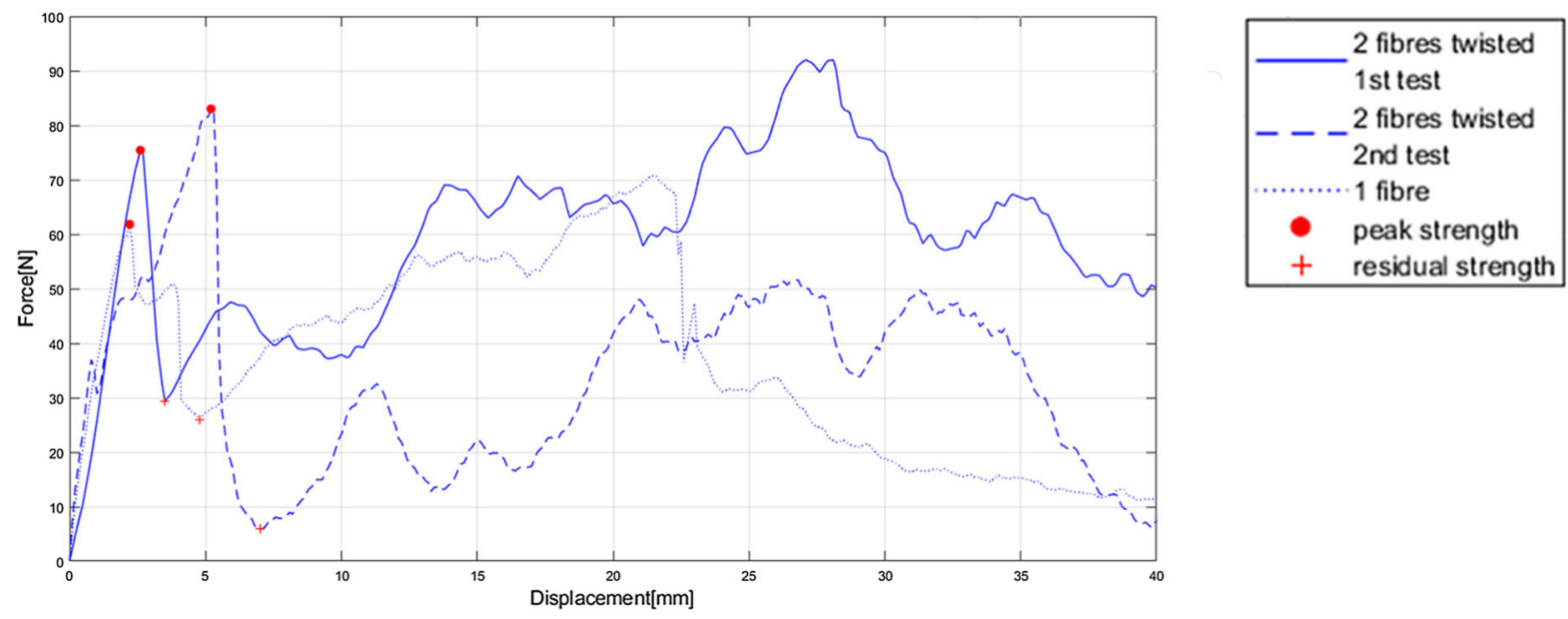

Fig. 9 Tests results for one fibre and two fibres twisted at a dry density of $2.00 \mathrm{Mg} / \mathrm{m}^{3}$, interface failure
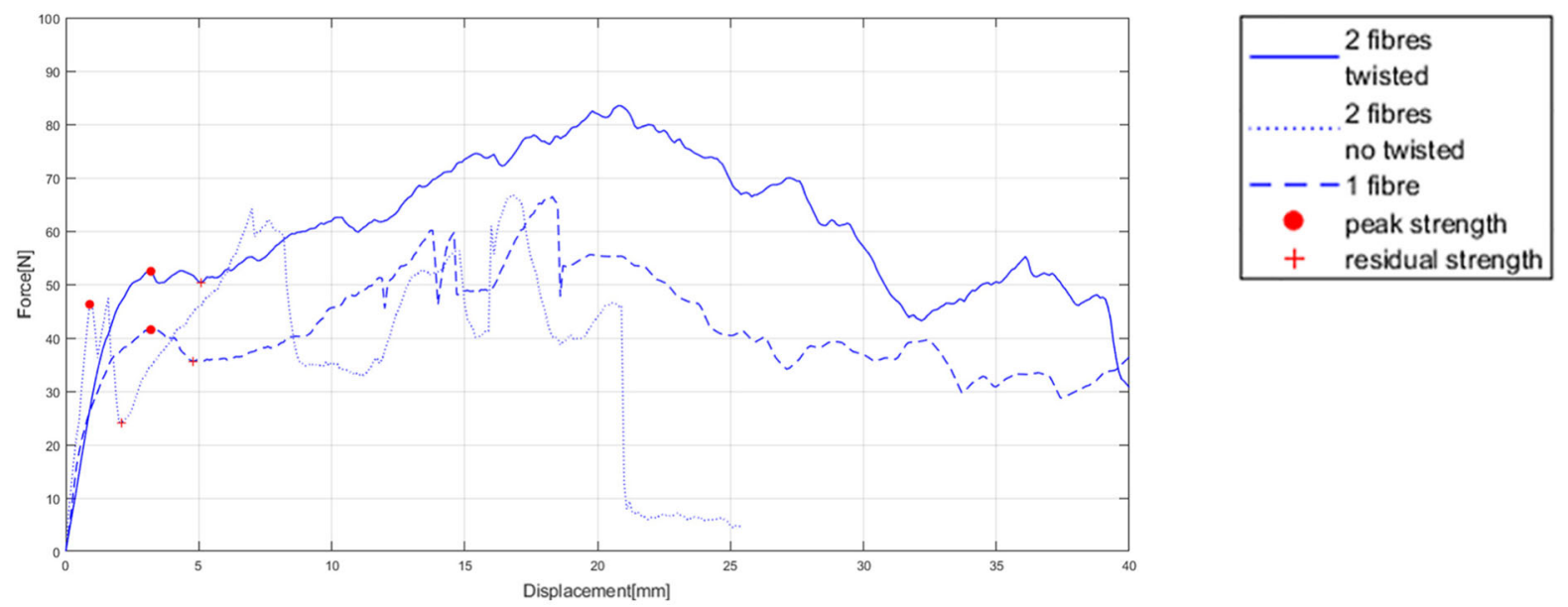

Fig. 10 Mean values results from tests with one fibre and two fibres twisted and untwisted at a dry density of $2.00 \mathrm{Mg} / \mathrm{m}^{3}, \mathrm{matrix}$ failure

the clay in the soil leading to weak bonding between fibre and soil and frictional resistance is probably also reduced, even though it could work as a protective coating improving fibre durability. Different results were obtained for fibres soaked in the strong or medium solutions. Figure 12 shows a peak load of almost $120 \mathrm{~N}$ for fibres soaked in the 50\% PVA solution, four times greater than samples tested without glue. Nevertheless, the residual strength is lacking. This behaviour might be explained by considering the fibre surface to be smooth once the bond is broken when the glue concentration is high. Samples with fibres soaked in the 20\% PVA solution achieve peak strength values slightly lower than the stronger solution case, but the residual strength improves, maybe supporting the smoothness conjecture. The matrix failure mode is not affected by the treatment with PVA glue so that the results are not reported here because they do not add significant information. This was expected because there are no additives in the rammed earth mix itself, so the bonding between particles is still the same. 

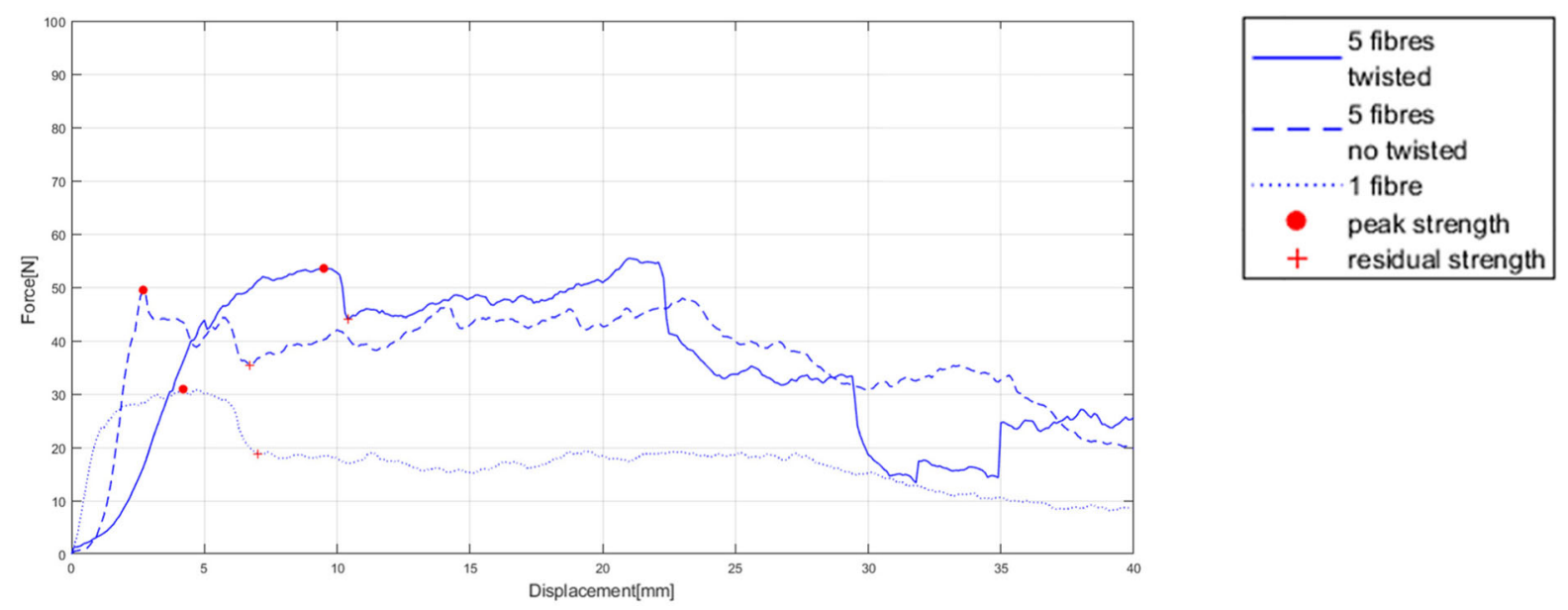

Fig. 11 Mean values results from tests with one fibre and five fibres twisted and untwisted at a dry density of $1.96 \mathrm{Mg} / \mathrm{m}^{3}$, interface failure
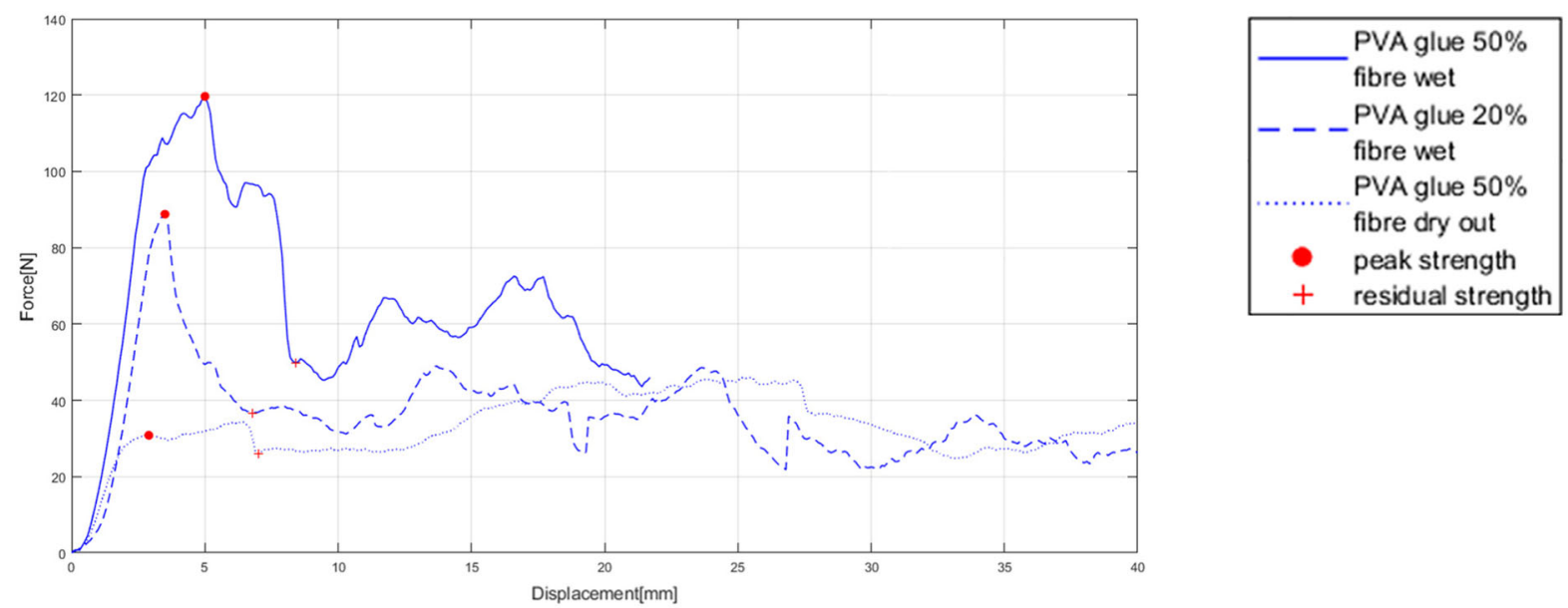

Fig. 12 Mean values results from tests with fibres soaked in the PVA solution at a dry density of $1.96 \mathrm{Mg} / \mathrm{m}^{3}$, interface failure

\subsection{Fibres treated with baking soda}

Fibres were soaked in two different baking soda solutions as described in detail in Sect. 4.3, then were left to dry out and used to make samples. The most remarkable result that emerges from the test data is that ductile behaviour increases for both matrix and interface failure mechanisms. This trend is illustrated in the plots in Figs. 13 and 14 respectively. The ductile behaviour is probably due to an improved frictional strength between fibres and matrix caused by a increase in fibre roughness after the alkali treatment. The peak strength reached by samples containing fibres soaked in a 50\% baking soda solution tested for the interface failure is low, probably due to a loss of structural integrity caused by the high percentage of the chemical compound that prevents the proper bonding with the soil. Tests on samples with fibres soaked in the $9 \%$ baking soda solution, in addition to the more ductile behaviour, also show higher peak strengths with special regard to the interface failure mode test, as shown in Fig. 14. 

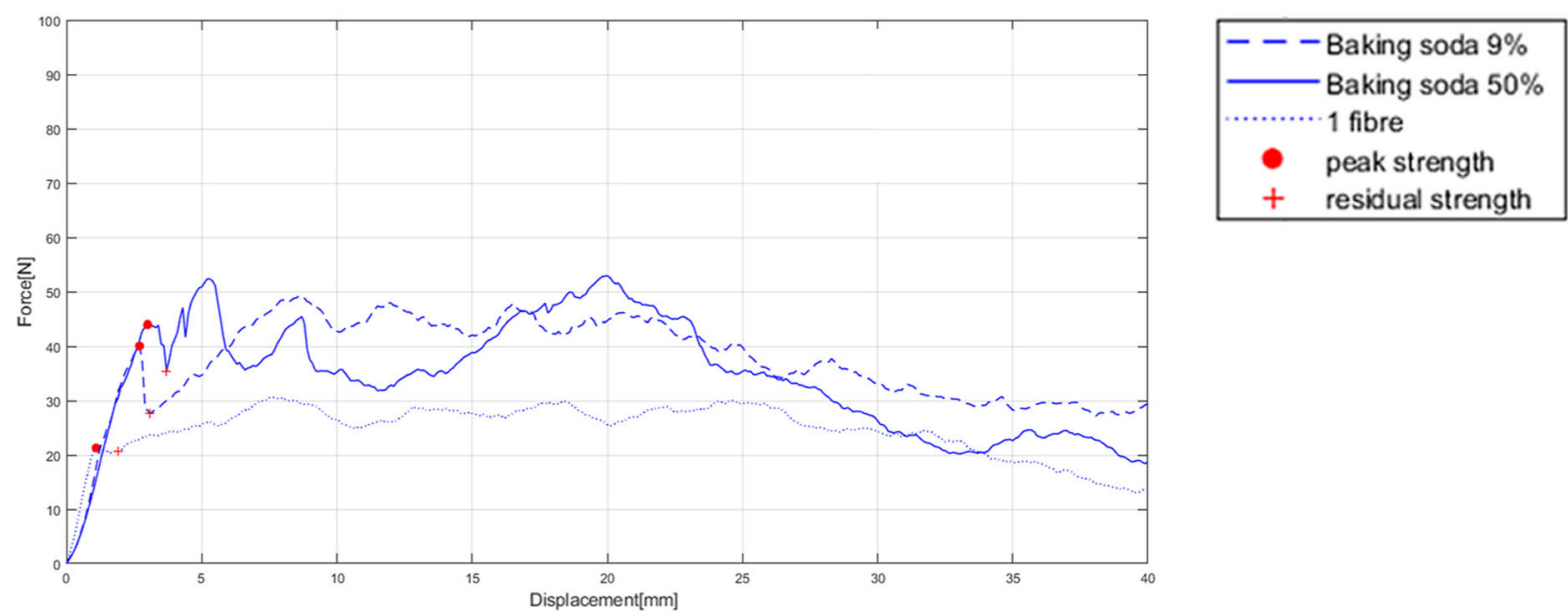

Fig. 13 Mean values results from tests with one fibre untreated and fibres soaked in baking soda solutions. Samples at a dry density of $1.96 \mathrm{Mg} / \mathrm{m}^{3}$, matrix failure
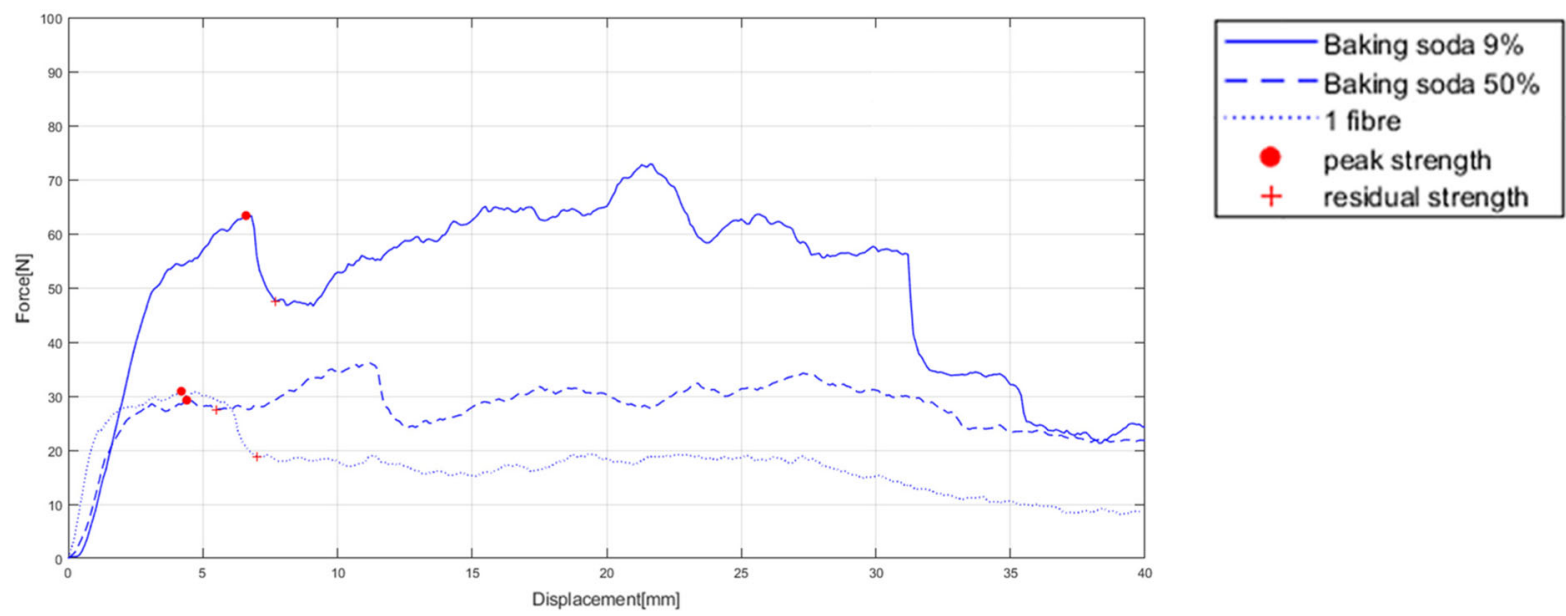

Fig. 14 Mean values results from tests with one fibre untreated and fibres soaked in baking soda solutions. Samples at a dry density of $1.96 \mathrm{Mg} / \mathrm{m}^{3}$, interface failure

\subsection{Summary of the main results}

Figure 15 compares results between samples with treated and untreated fibres. Samples with fibres soaked in the $50 \%$ PVA glue solution and manufactured wet reach the highest peak load of about $120 \mathrm{~N}$, which is an increase of $400 \%$ compared to samples with untreated fibres. The peak load achieved by samples with fibres treated with baking soda solution is $63 \mathrm{~N}$, lower than fibres treated with PVA glue but still more than twice the value for samples with untreated fibres. Moreover the residual strength values are the highest experienced in all the tests. The main results in terms of peak, PS, and residual strength, RS, average values, peak strength coefficient of variation, PS-c, and pull-out energy at residual strength, RE, for each of the investigated parameters are summarized in Tables 3 and 4 considering both interface and matrix failure modes, respectively. The experimental results imply that the mechanical properties of the composite earthen construction material improve if the bond between fibres and matrix fully allows for the stress transfer between the two components. This finding suggests that special care should be given in ensuring a 

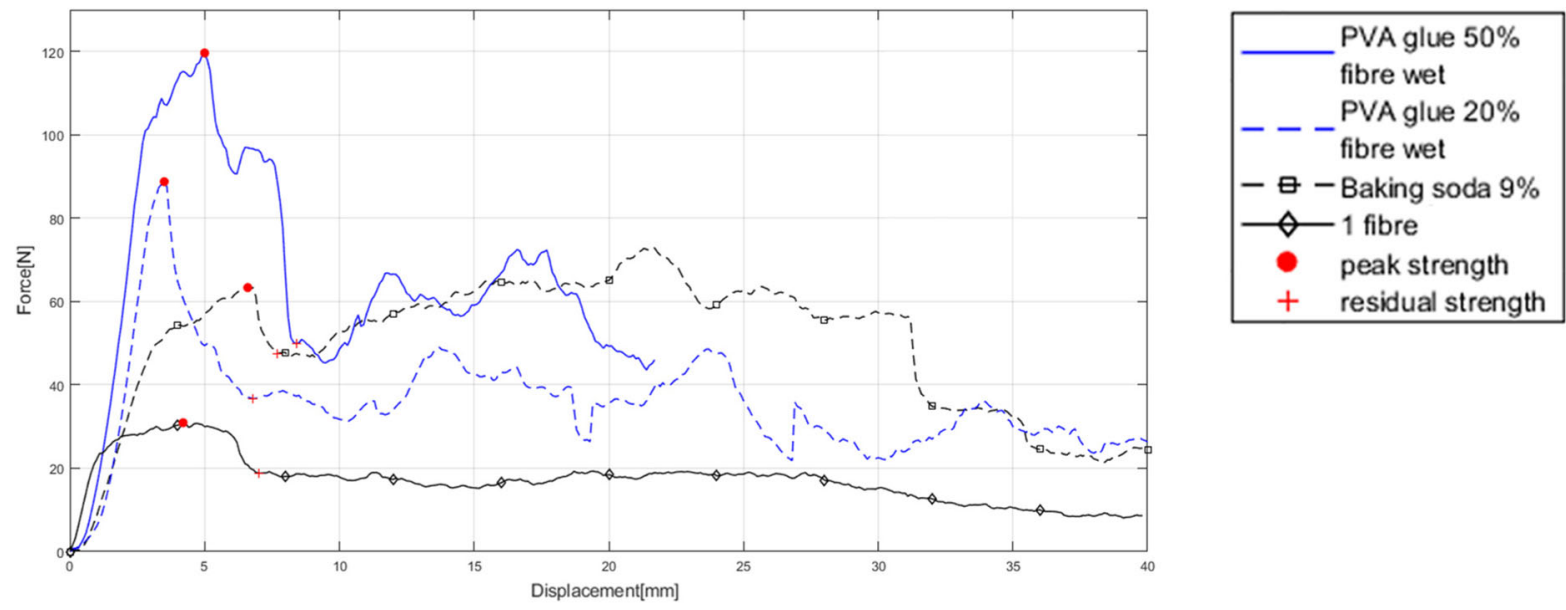

Fig. 15 Mean values results from tests with treated and untreated fibres. Samples at a dry density of $1.96 \mathrm{Mg} / \mathrm{m}^{3}$, interface failure

Table 3 Summary of the peak, PS, and residual strength, RS, average values, peak strength coefficient of variation, PS-c, and pull-out energy at residual strength, $\mathrm{RE}$, for the interface failure mode

\begin{tabular}{lcclc}
\hline Parameter & PS & PS-c & RS & RE \\
& N & & Nmm \\
\hline One fibre 2.00 dd & 61.83 & 0.29 & 25.99 & 195.36 \\
One fibre 1.96 dd & 30.91 & 0.33 & 18.81 & 177.60 \\
Two fibres no twisted 1.96 dd & 64.41 & 0.30 & 41.39 & 196.33 \\
Two fibres twisted 2.00 dd & 79.24 & 0.07 & 17.64 & 226.09 \\
Five fibres no twisted 1.96 dd & 49.47 & 0.14 & 35.38 & 216.48 \\
Five fibres twisted 1.96 dd & 53.53 & 0.10 & 44.03 & 361.71 \\
50\% PVA glue fibres dry out 1.96 dd & 30.81 & 0.40 & 26.06 & 181.89 \\
50\% PVA glue fibres wet 1.96 dd & 119.64 & 0.25 & 49.81 & 662.34 \\
20\% PVA glue fibres wet 1.96 dd & 88.75 & 0.27 & 36.68 & 295.95 \\
50\% Baking soda 1.96 dd & 29.27 & 0.20 & 27.41 & 121.63 \\
9\% Baking soda 1.96 dd & 63.34 & 0.27 & 47.45 & 326.48 \\
\hline
\end{tabular}

proper distance between multiple fibres when con-

Table 4 Summary of the peak, PS, and residual strength, RS, average values, peak strength coefficient of variation, PS-c, and pull-out energy at residual strength, RE, for the matrix failure mode

\begin{tabular}{lllll}
\hline Parameter & PS & PS-c & RS & RE \\
& N & & Nmm \\
\hline One fibre 2.00 dd & 41.56 & 0.33 & 35.60 & 161.74 \\
One fibre 1.96 dd & 21.29 & 0.14 & 20.63 & 29.93 \\
Two fibres no twisted & 46.23 & 0.32 & 24.03 & 68.43 \\
$\quad$ 2.00 dd & & & & \\
Two fibres twisted 2.00 dd & 52.43 & 0.25 & 50.30 & 213.67 \\
50\% Baking soda 1.96 dd & 43.98 & 0.10 & 35.43 & 98.66 \\
9\% Baking soda 1.96 dd & 40.05 & 0.44 & 27.68 & 70.74 \\
\hline
\end{tabular}

structing full scale earthen structures using fibres. In this case proper bonding would be guaranteed by having a layer of earth surrounding each fibre. Fibre content and mixture procedures should avoid fibre bunching as the study here suggests there is little benefit in having multiple fibre sets. The helical shape of twisted fibres also improve the bonding as shown by test results in Sect. 5.3 although to ensure this in practice would require pre-treatment of the fibres before incorporation into the mix, which may be costly or problematic. As far as the treatments are concerned, both PVA glue and alkali treatments improve the pullout strength of the fibres and hence would improve the mechanical properties of the composite material in 
practice. This would suggest that the manufacturing procedure should involve treatment of fibres just prior to incorporation in the soil mix.

\section{Conclusion}

This work has investigated the pull-out strength of jute fibres incorporated into a soil mixture that might be used for earthen construction. Tests were carried varying the number of fibres in a single sample, or their treatment in terms of pre-twisting or application of chemical treatments changing solution concentrations. The chemical treatments were chosen to be both widely available and of low hazard of harm in their use (looking ahead to practical application). Results showed that significant increases in pull-out strengths can be gained using the chemical treatments and that twisting can also improve behaviour due to the introduction of a more complex surface geometry to the fibres, increasing frictional resistance. As far as chemical treatment goes, the PVA glue has the largest influence on the peak load values due to a good bond developing between glue and clay. Reducing the PVA glue concentration from 50 to $20 \%$, the peak strength slightly decreases but the residual strength is maintained, and this could be a viable option in practice, balancing property enhancement with cost. The paper highlights that interaction between fibre and earthen construction materials is a topic with very little published research and clearly further work is essential to understand in depth the interaction characteristics. Therefore, the experimental test results reported in this work represent important novel information that starts to fill the gap. Additional tests could be carried out adding glue into the soil mix to study the effect on the peak and residual strength for the matrix failure mode. Durability is also a key issue that must be investigated, with particular reference to the influence on jute fibres subjected to alkali treatments and there are questions as to the longevity of natural fibres and chemical treatments. Tests on larger samples, designing different equipment and test procedures, are also needed to confirm the insitu behaviour of full scale earthen constructions.

Acknowledgements This research was carried out while the first author was a visiting student at Durham University, supported by the EC-funded ERASMUS programme. The assistance of technical staff in the Department of Engineering Civil Engineering Laboratory is gratefully acknowledged.

Funding Open access funding provided by Università degli Studi di Perugia within the CRUI-CARE Agreement.

\section{Declarations}

Conflict of interest The authors declare that they have no conflict of interest.

Open Access This article is licensed under a Creative Commons Attribution 4.0 International License, which permits use, sharing, adaptation, distribution and reproduction in any medium or format, as long as you give appropriate credit to the original author(s) and the source, provide a link to the Creative Commons licence, and indicate if changes were made. The images or other third party material in this article are included in the article's Creative Commons licence, unless indicated otherwise in a credit line to the material. If material is not included in the article's Creative Commons licence and your intended use is not permitted by statutory regulation or exceeds the permitted use, you will need to obtain permission directly from the copyright holder. To view a copy of this licence, visit http://creativecommons.org/licenses/by/4.0/.

\section{References}

1. Minke G (2006) Building with earth. Birkhãuser-Publishers for Architecture, Design and Technology of a Suistanable Architecture

2. Jaquin P (2008) Analysis of historic rammed earth construction. Ph.D. Dissertation, Durham University, UK

3. Morton T, Little B (2001) Building with earth in Scotland: innovative design and sustainability. Executive Central Research Unit, Edinburgh

4. Easton D (2007) The Rammed Earth House. Chelsea Green Publishing Company, White River Junction

5. Augarde CE, Beckett CT, Smith JC, Corbin AJ (2016) Challenges in treating earthen construction materials as unsaturated soils. In: E3S web of conferences, vol 9, EDP Sciences, p 03002

6. Smith JC, Augarde C (2014) Optimum water content tests for earthen construction materials. Proc Inst Civil Eng Constr Mater 167:114-123

7. Walker P, Keable R, Martin J, Maniatidis V (2005) Rammed earth: design and construction guidelines. BRE Bookshop, Watford

8. Jaquin P, Augarde C (2012) Earth building: History, science and conservation. IHS BRE Press, Bracknell

9. Readle D (2013) The bonding of fibrous material in rammed earth. MEng Dissertation, Durham University, UK

10. Coghlan S (2014) Bonding in fibre-reinforced earthen construction materials. MEng Dissertation, Durham University, UK

11. Readle D, Coghlan S, Smith JC, Corbin A, Augarde CE (2016) Fibre reinforcement in earthen construction materials. Proc Inst Civil Eng Constr Mater 169(2016):252-260 
12. Morton T (2010) Earth masonry: design and construction guidelines (Ep 80). IHS BRE Press, Bracknell

13. Rathod RSB, Reddy BV (2021) Strength and stress-strain characteristics of fibre reinforced cement stabilised rammed earth. Mater Struct 54:1-13

14. Chang I, Prasidhi A, Cho GC (2016) Durability improvement of earth walls using biopolymer treated Korean residual soil

15. Muguda S, Booth SJ, Hughes PN, Augarde CE, Perlot C, Bruno AW, Gallipoli D (2017) Mechanical properties of biopolymer-stabilised soil-based construction materials. Géotech Lett 7:309-314

16. Harichane K, Ghrici M, Kenai S (2012) Effect of the combination of lime and natural pozzolana on the compaction and strength of soft clayey soils: a preliminary study. Environ Earth Sci 66:2197-2205

17. ArchDaily (2016) Vineyard house/blaanc, 12 January

18. Dezeen (2014) Herzog \& de meuron uses rammed earth to create countryside herb centre for ricola, 7 July

19. Ling HI, Liu H, Kaliakin VN, Leshchinsky D (2004) Analyzing dynamic behavior of geosynthetic-reinforced soil retaining walls. J Eng Mech 130:911-920

20. Bordoloi S, Garg A, Sekharan S (2017) A review of physiobiochemical properties of natural fibers and their application in soil reinforcement. Adv Civil Eng Mater 6:323-359

21. Tang C-S, Shi B, Zhao L-Z (2010) Interfacial shear strength of fiber reinforced soil. Geotext Geomembr 28:54-62

22. Yetimoglu T, Salbas O (2003) A study on shear strength of sands reinforced with randomly distributed discrete fibers. Geotext Geomembr 21:103-110

23. Ismail S, Yaacob Z (2011) Properties of laterite brick reinforced with oil palm empty fruit bunch fibres. Pertanika J Sci Technol 19:33-43

24. Maniatidis V, Walker P (2003) A review of rammed earth construction. Innovation Project "Developing Rammed Earth for UK Housing”, Natural Building Technology Group. University of Bath, Department of Architecture \& Civil Engineering

25. Piattoni Q, Quagliarini E, Lenci S (2011) Experimental analysis and modelling of the mechanical behaviour of earthen bricks. Constr Build Mater 25:2067-2075

26. Villamizar MCN, Araque VS, Reyes CAR, Silva RS (2012) Effect of the addition of coal-ash and cassava peels on the engineering properties of compressed earth blocks. Constr Build Mater 36:276-286

27. Eko RM, Offa ED, Ngatcha TY, Minsili LS (2012) Potential of salvaged steel fibers for reinforcement of unfired earth blocks. Constr Build Mater 35:340-346

28. Cheah JSJ, Walker P, Heath A, Morgan TKKB (2012) Evaluating shear test methods for stabilised rammed earth. Proc Inst Civil Eng Constr Mater 165:325-334
29. Corbin A, Augarde C (2015) Investigation into the shear behaviour of rammed earth using shear box tests. In: Proceedings of the first international conference on bio-based building materials, pp 93-98

30. Millogo Y, Morel J-C, Aubert J-E, Ghavami K (2014) Experimental analysis of pressed adobe blocks reinforced with Hibiscus cannabinus fibers. Constr Build Mater 52:71-78

31. Houben H, Guillaud H, Hall BB (1994) Earth construction: a comprehensive guide. Intermediate Technology Publications, London

32. Marandi S, Bagheripour M, Rahgozar R, Zare H et al (2008) Strength and ductility of randomly distributed palm fibers reinforced silty-sand soils. Am J Appl Sci 5:209-220

33. Schroeder H, Schnellert T, Heller T, Sowoidnich T (2005) Moisture transfer and change in strength during construction of rammed earth. In: Proceedings of Kerpic 05: Living in Earthen Cities, Istanbul Techinical University, Turkey

34. Tang C, Shi B, Gao W, Chen F, Cai Y (2007) Strength and mechanical behavior of short polypropylene fiber reinforced and cement stabilized clayey soil. Geotext Geomembr 25:194-202

35. Ghavami K, Toledo Filho RD, Barbosa NP (1999) Behaviour of composite soil reinforced with natural fibres. Cement Concr Compos 21:39-48

36. Nam TH, Ogihara S, Nakatani H, Kobayashi S, Song JI (2012) Mechanical and thermal properties and water absorption of jute fiber reinforced poly (butylene succinate) biodegradable composites. Adv Compos Mater 21:241-258

37. Zamri MH, Akil HM, Bakar AA, Ishak ZAM, Cheng LW (2012) Effect of water absorption on pultruded jute/glass fiber-reinforced unsaturated polyester hybrid composites. J Compos Mater 46:51-61

38. Smith J, Augarde C (2013) A new classification for soil mixtures with application to earthen construction. Techical Report ECS-TR 2013/04. Durham University, Engineering and Computing Sciences

39. Hall M, Djerbib Y (2004) Rammed earth sample production: context, recommendations and consistency. Constr Build Mater 18:281-286

40. Maniatidis V, Walker P, Heath A, Hayward S (2007) Mechanical and thermal characteristics of rammed earth. In: International Symposium on Earthen Structures, University of Bath

Publisher's Note Springer Nature remains neutral with regard to jurisdictional claims in published maps and institutional affiliations. 